\title{
CT-Guided Percutaneous Lumbar Ligamentum Flavum Release by Needle Knife for Treatment of Lumbar Spinal Stenosis: A Case Report and Literature Review
}

This article was published in the following Dove Press journal: Journal of Pain Research

Xinyue Zhu, ${ }^{1,2}$ Zuyun Qiu, (D) ${ }^{1,3}$ Zixiang Liu, ' Yifeng Shen, ${ }^{4}$ Qiaoyin Zhou, ${ }^{5}$ Yan Jia, ${ }^{1,3}$ Xiaojie Sun, 1,3 Shiliang Li'

'Department of Acupuncture and Moxibustion, China-Japan Friendship Hospital, Beijing,

People's Republic of China; ${ }^{2}$ Department of Rehabilitation Medicine, Beijing First Hospital of Integrated Chinese and Western Medicine, Beijing, People's Republic of China; ${ }^{3}$ Graduate School, Beijing University of Traditional Chinese Medicine, Beijing, People's Republic of China; ${ }^{4}$ Department of Traditional Chinese Surgery, Hospital of Chengdu University of Traditional Chinese Medicine, Chengdu, Sichuan, People's Republic of China; ${ }^{5}$ Chinese Medicine College, Fujian University of Traditional Chinese Medicine, Fuzhou, Fujian, People's Republic of China

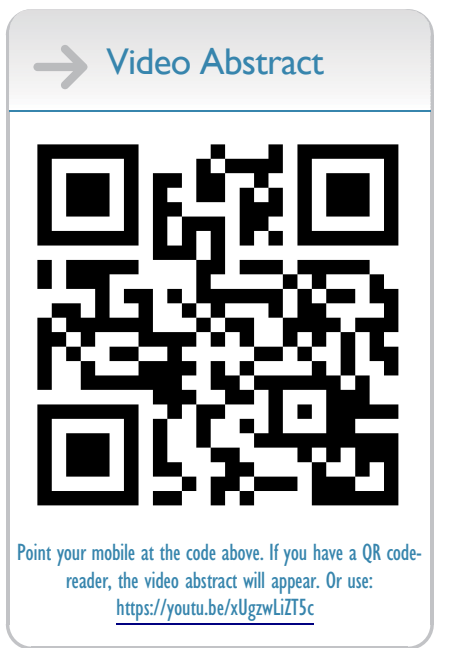

Correspondence: Shiliang $\mathrm{Li}$ Department of Acupuncture and Moxibustion, China-Japan Friendship Hospital, 4 Sakura Garden Road, Beijing 100029, People's Republic of China Tel +86-I35-8I77-6268

Email zrlishiliang@I63.com
Case: The patient was a 62-year-old woman presenting with low-back pain of 3 years' duration and numbness in the right leg.

Diagnosis: She was diagnosed with lumbar spinal stenosis based on combined magnetic resonance imaging, physical examination, and symptoms.

Interventions: Treatment with computed tomography-guided percutaneous release of the ligamentum flavum was delivered.

Outcomes: Relief of symptoms immediately after treatment and complete resolution of symptoms after 1 month were achieved. At follow-up, there was no recurrence of symptoms after 2, 4, 6, and 12 months.

Conclusion: Lumbar ligamentum flavum hypertrophy is an important cause of degenerative lumbar spinal stenosis. The hypertrophic ligamentum flavum can directly compress the spinal canal, squeeze the cauda equina, and simultaneously lead to a reduction in the anteroposterior diameter of the intervertebral foramen, which compresses the nerve roots and causes numbness and other symptoms of lower extremities. In clinical practice, doctors should combine imaging findings with patient symptoms for diagnosis and an individualized treatment plan for each patient with lumbar spinal stenosis, and conduct gradual stepwise treatment using conservative minimally invasive surgery to prevent excessive surgery.

Keywords: CT guidance, ligamentum flavum, lumbar spinal stenosis, minimally invasive surgery, needle knife

\section{Introduction}

Lumbar spinal stenosis (LSS) refers to changes in morphology and tissue structure of the lumbar vertebrae and soft tissue (eg, vertebral body, facet joint, lamina, ligamentum flavum (LF), intervertebral disk) caused by various factors that results in stimulation or compression of the central spinal canal and lateral crypts and nerve pores at the nerve roots or cauda equina, thereby causing several clinical symptoms. Common causes include degenerative, congenital, iatrogenic, and traumatic factors, isthmic spondylolisthesis, metabolic and endocrine diseases, infectious diseases, tumors, and cartilage diseases. ${ }^{1}$ Degenerative LSS is a common disease of the spine that causes severe pain, disability, and dysfunction. ${ }^{2-6}$ Prevalence of LSS in the general population is estimated at $9 \%$ and up to $47 \%$ in people over the age of 60 years. ${ }^{7}$ LSS is the most common indication for spine surgery in patients aged over 65 years. ${ }^{8}$

Lumbar LF hypertrophy (LFH) is a clinical pathological phenomenon considered one of the causes of LSS. The LF forms an aspect of the posterior wall of the spinal 
canal, and hypertrophy of each part of the LF can directly compress the spinal canal and cauda equina. Moreover, since the ligamentum flavum also forms part of the posterior edge of the intervertebral foramen, under hypertrophic conditions it causes a decrease in the width of the intervertebral foramen, which results in venous congestion and high pressure around the nerve roots, thereby leading to the limb symptoms. Currently, most clinicians recommend surgery for the treatment of LSS; however, most patients with LSS refuse surgery, because of surgical risks, complications, low surgical tolerance, and financial burden. Minimally invasive spinal surgeries have been gaining popularity recently, because of decreased iatrogenic trauma, comparable clinical outcomes, and shortened hospitalization. ${ }^{9-11}$ To the best of our knowledge, this is the first study to report success with computed tomography (CT)-guided percutaneous release of the lumbar ligamentum in a patient with LSS.

\section{Case Report}

A 62-year-old woman sought medical help in our department for severe low-back pain of 3 years' duration with accompanied numbness of the right leg. Initially, the patient had no obvious cause of low-back pain and did not go to the hospital for treatment. Application of an analgesic plaster achieved poor curative effect. Subsequently, the back pain gradually worsened, and numbness of the right leg developed at the same time and progressed over the course of 3 years. She visited our department for treatment on March $9^{t}$, 2018. She complained that if she sat for more than 2 hours or walked more than $300 \mathrm{~m}$, she experienced low-back pain accompanied by numbness at the front side of the right thigh and lateral calf, and relief of symptoms when she was lying down or bending forward. However, these symptoms seriously affected her daily life and activities.

The patient was subjected to a straight leg-raising test, and the result showed that the right side was positive at $70^{\circ}$ and the left side negative. There was no obvious tenderness at the patient's waist. Patient evaluation was performed using the VAS and Japanese Orthopaedic Association (JOA) scores before and after treatment. In the results Before treatment, scores of VAS of 6 points and JOA of 10 points were obtained. Based on these findings, she was recommended to undergo lumbar magnetic resonance imaging (MRI) to assist in diagnosis. In MRI results (Figure 1), lumbar curvature was normal and anterior and posterior margins of multiple vertebral bodies rough and sharp. On the $T_{2}$-weighted image scan, presence of multiple signals of the intervertebral disk, bulging of the intervertebral disk at L2/3,
$\mathrm{L} 3 / 4$, and L4/5, protrusion of the intervertebral disk at L5/ $\mathrm{S} 1$, dural sac compression at corresponding levels, LFH at L2/3 L3/4, and L4/5, and LSS were observed.

Before the operation, positioning and labeling of L2/3, $\mathrm{L} 3 / 4$, and L4/5 with the positioning grid was performed under CT guidance. Subsequently, the CT scan was used to measure the bilateral thickness of the LF at L2/3, L3/4, and $\mathrm{L} 4 / 5$. The angle and depth of the needle on the left and right sides of the L2/3, L3/4, and L4/5 segments were measured on the CT-scan image and the needle-insertion point marked on the body surface (Figure 2). For routine surgical disinfection, $2 \%$ lidocaine was injected into each of the facet joints. The facet joint was punctured with the needle knife, the direction and depth of the needle adjusted to the edge of the lower facet, and the position reconfirmed by CT scan (Figure 3). Next, the needle knife was used to cut the attachment point of the LF to about $2-3 \mathrm{~mm}$ depth and pressed for 10 minutes, and the patient was instructed to maintain a supine position for 6 hours. After surgery, there was a small amount of hemorrhage, no infection, ecchymoma, headache, dizziness, or numbness, and pain and weakness of the lower limbs and other complications (Figure 4). Then, the thickness of the bilateral LF at L2/3, L3/4, and L4/5 was measured (Table 1). In the straight leg-elevation test performed immediately after the operation, negative results on the VAS ( 0 points) and JOA (10 points) were obtained at both sides. She was followed up after treatment at 1, 2, 4, 6, and 12 months (Table 2). In the results of follow-up evaluation at 12 months, VAS of 0 points and JOA of 29 points were obtained. The patient's waist and leg pain had completely resolved.

\section{Literature Review}

We searched for relevant studies in PubMed using the terms "lumbar ligamentum flavum hypertrophy", "lumbar ligamentum flavum thickening", and "lumbar ligamentum flavum calcification". As a result, 136 relevant citations from 1956 to September 2018 were retrieved. Only fulltext articles of case reports in English were reviewed. Among these, the main focus was to study the pathological and physiological mechanisms of hypertrophy of the ligamentum flavum, and few reports of clinical cases were present. For each case, specific details extracted were epidemiological characteristics, chronological details, chief complaint at presentation, symptoms, imaging findings, and treatment.

LFH is considered a major cause of (LSS), ${ }^{12}$ and can also lead to lateral recess stenosis and foramen stenosis, 

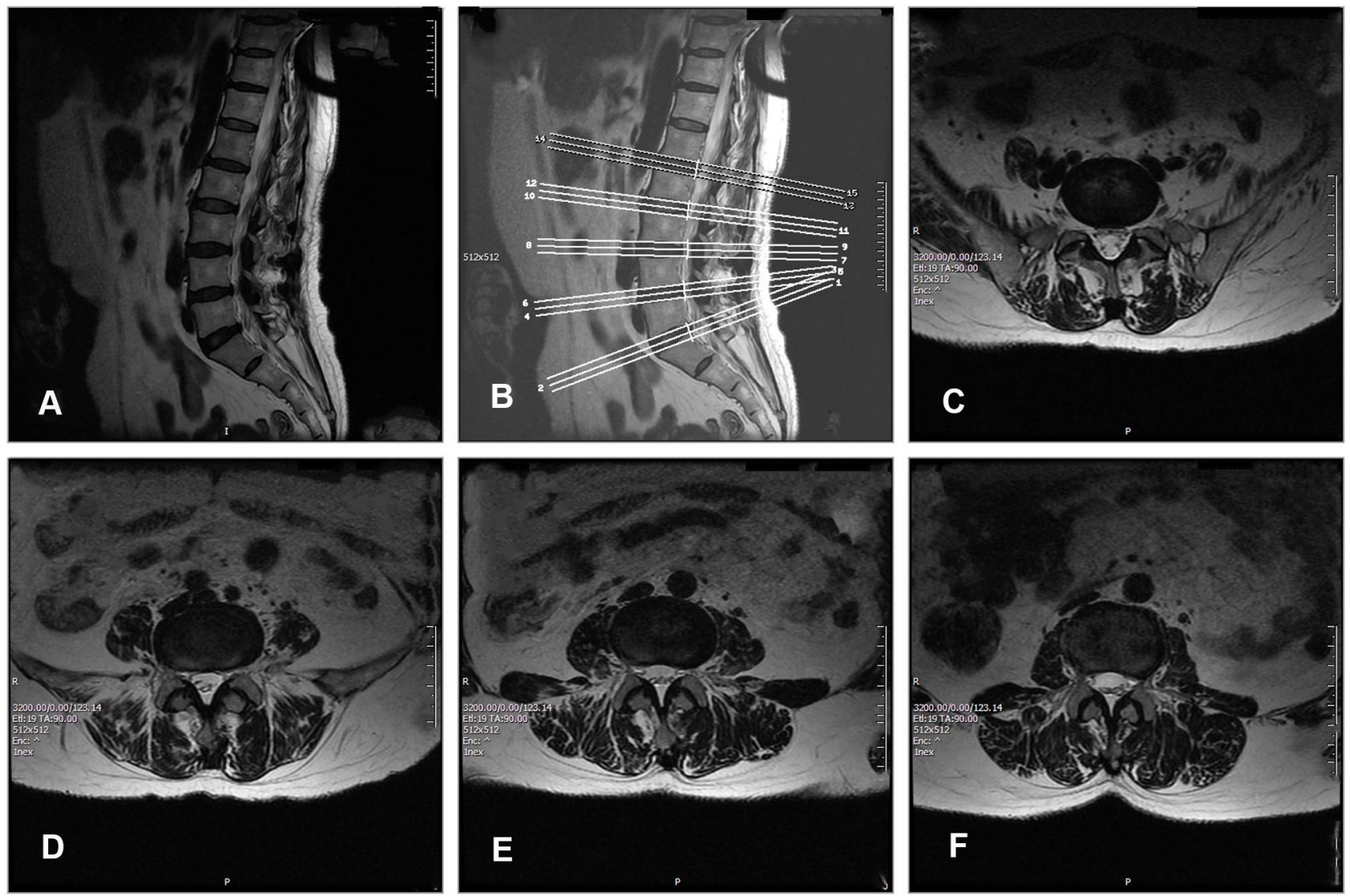

Figure I (A) Normal lumbar curve and alignment; (B) sagittal location map; (C) L5/SI disk herniation; (D) L4/5 ligamentum flavum hypertrophy; (E) L3/4 ligamentum flavum hypertrophy; (F) L2/3 ligamentum flavum hypertrophy.

thereby leading to simultaneous compression of the spinal cord and nerve roots. ${ }^{13}$ Age-related increases in disk degeneration combined with continuous lumbar segmental flexion-extension motion lead to development of LFH. ${ }^{14}$ Saito et al reported that mechanical stress was a causative factor for LFH and stressed the importance of macrophage infiltration in the progression of LFH via stimulation of collagen production. ${ }^{15}$ Chelladurai et al reported that there was no significant difference in LFH between the two sides and sex. ${ }^{16}$ Currently, there are two treatment approaches in patients with thickening of the LF: operative treatment and nonoperative treatment. For operative treatment, several methods of excision of the LF, eg, decompressive laminectomy and minimally invasive decompression, are performed under general principles of full decompression and spinal stability. For nonsurgical treatment, targeted drugs are mainly used in oral or injectable form, and in cases of poor effectiveness are indicated for surgery. Most investigators consider that operative treatment should be the first treatment of choice. ${ }^{17}$
In the literature review, 540 patients were identified (Table 3). Patient ages were 33-79 years, with an average of approximately 54.2 years. Among patients identified with LSS, $166(30.7 \%)$ were women and 374 (69.3\%) were men. Operative treatment $(71.6 \%)$ was the main option, including excision of the LF (35.9\%), decompressive laminectomy $(8.1 \%)$, and minimally invasive decompression $(27.6 \%)$. Nonoperative treatment (28.4\%) comprised epidural steroid injections. Operative treatment is more effective in relieving clinical symptoms caused by LFH than nonsurgical treatment.

\section{Discussion}

Lumbar degenerative diseases include lumbar disk herniation, facet-joint hyperplasia, and LFH, which can lead to LSS. Because the LF covers the lateral posterior aspect of the lumbar spinal canal, hyperplasia is considered one of the most important causes of LSS. Li et al reported that degeneration of the intervertebral disk and facet joints leads to abnormal stress in the LF, which promotes hypertrophy of the LF. ${ }^{18}$ The stress 

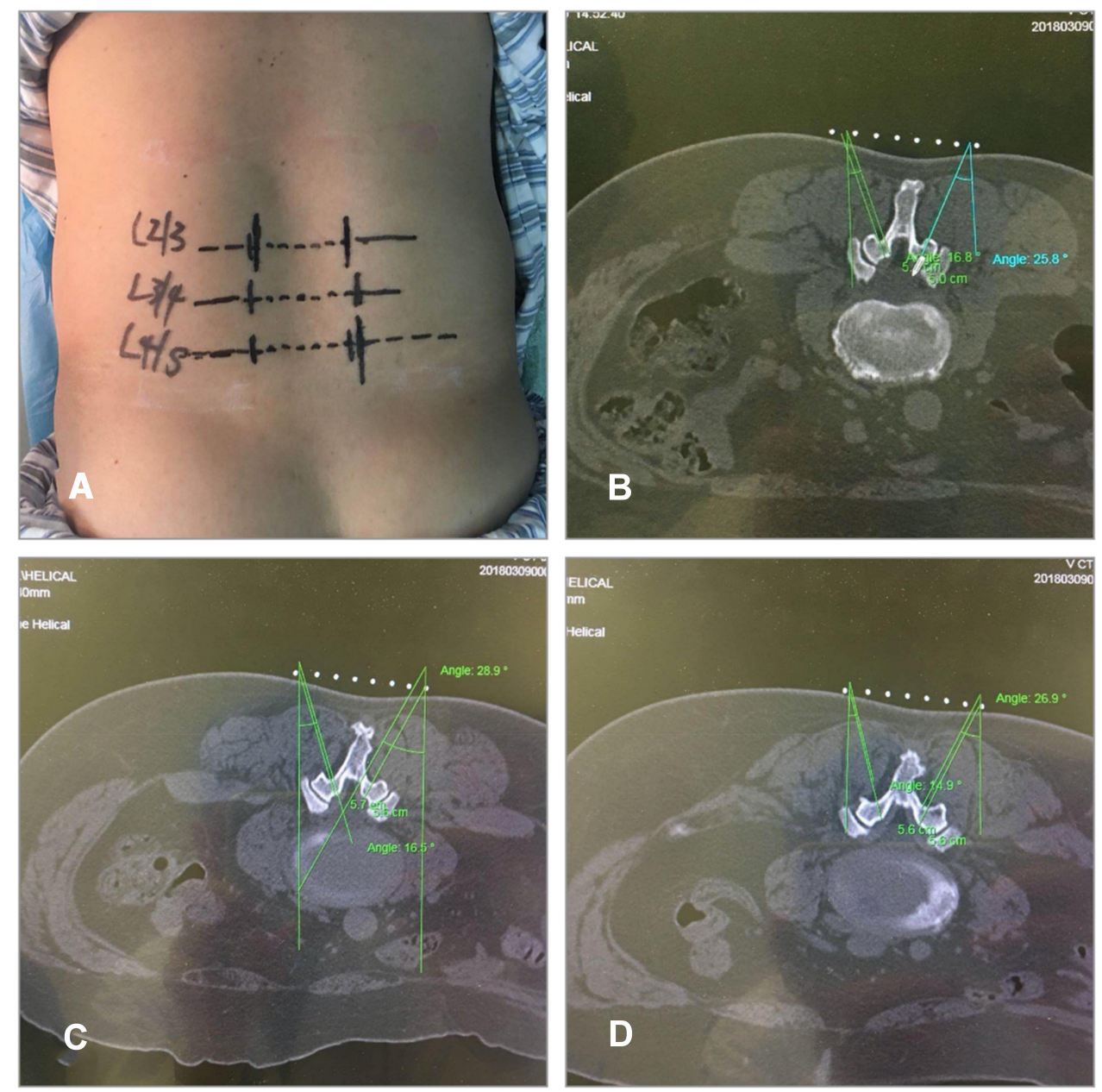

Figure 2 (A) Needle-knife insertion point on the body surface: (B) angle and depth for L2/3; (C) angle and depth for L3/4; (D) angle and depth for L4/5.

abnormality causes lumbar spine instability and a consequent repeating cycle of local injury and repair, which results in hypertrophy and even ossification of the LF. The lumbar ligamentum is divided into two parts: the medial part, located between the lamina, which comprises an aspect of the posterior wall and posterior lateral wall of the central spinal canal, and the lateral part, which extends outward and merges with the intervertebral facet-joint capsule with gradual thinning, and forms aspects of the central part, posterior wall of the spinal canal, lateral recess, and intervertebral foramen. ${ }^{19}$ Due to these anatomical features, lumbar LFH causes stenosis of the spinal canal, and through merging with the lateral crypts and intervertebral foramen stenosis, results in simultaneous compression of the spinal cord and nerve roots. ${ }^{13}$ Studies have shown that lumbar LFH often coincides with lumbar disk herniation and facet-joint degeneration, and hence these patients present typical clinical symptoms of lowback pain and intermittent claudication, ${ }^{20}$ similar to those in our patients. In MRI and CT images acquired in our study, LFH of the lumbar spine was accompanied by lumbar disk herniation and degeneration of the facet joints at the corresponding segments.

A recent report of the North American Spinal Association indicated that a diagnosis of LSS should made in patients manifesting symptoms of the hip or lower-extremity pain, with or without lumbar pain, due to reduced space available for the nerves and blood vessels within the spinal canal. However, the diagnostic method for LSS still lacks a gold standard and is currently based on a combination of medical history, physical examination, and imaging studies. ${ }^{21}$ In our patient, the performance of imaging contributed less to the diagnosis than the symptoms. Nevertheless, the combined results of medical history, physical examination, and imaging enabled the diagnosis of LSS.

The current treatment approach for LF thickening includes operative and nonoperative treatment. Operative treatment includes such procedures as excision of the LF, 

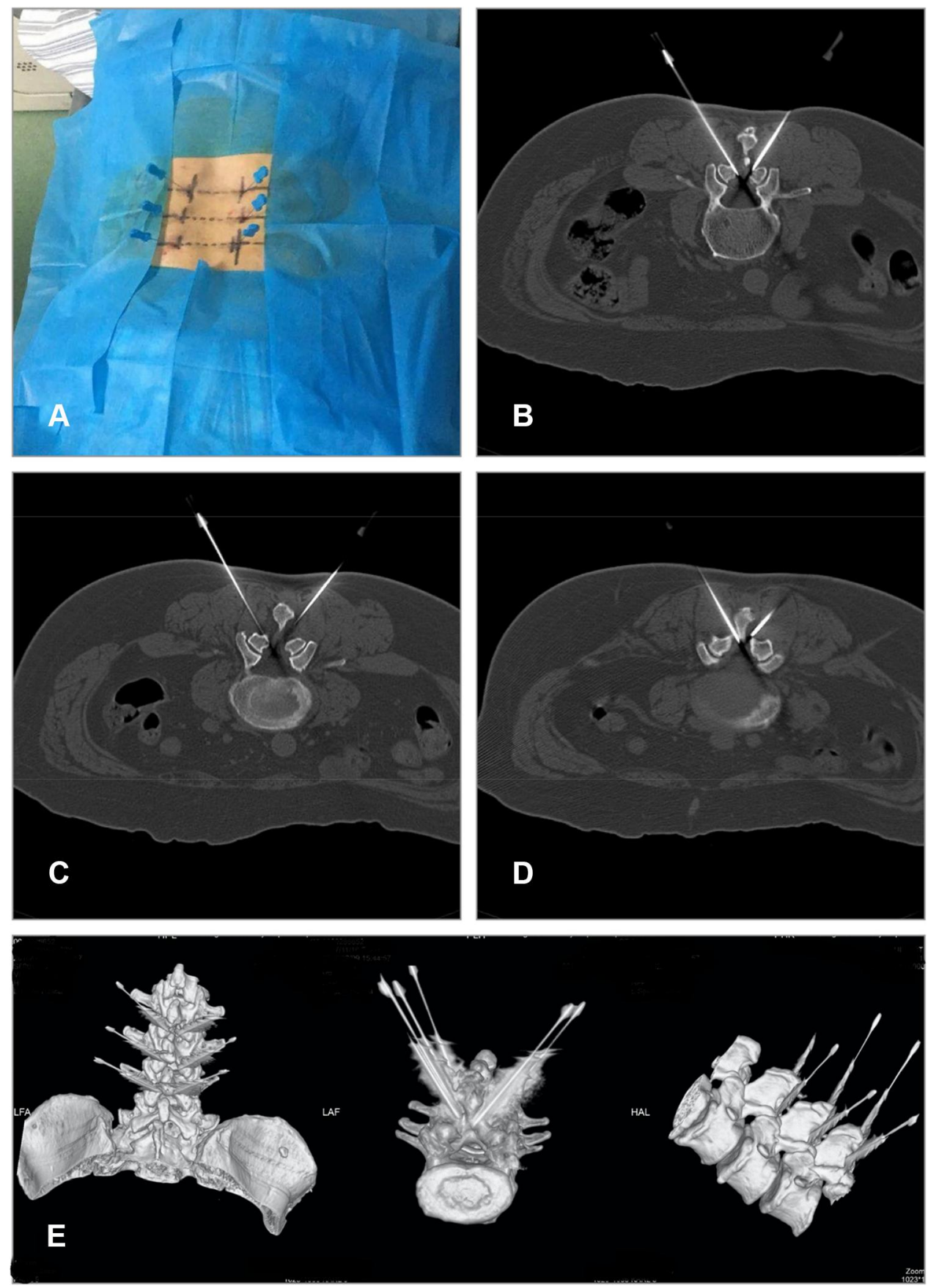

Figure 3 (A) Needle-knife position on the body surface: (B) for L2/3; (C) for L3/4; (D) for L4/5. (E) 3-D reconstruction.

decompressive laminectomy, and minimally invasive decompression. Several operative methods are available, but in general surgeons follow the guiding principles of full decompression and spinal stability. Nonsurgical treatment mainly involves targeted drugs administered orally or by injection, and operative treatment is indicated in nonresponders. ${ }^{17}$ Most investigators have recommended operative treatment as first treatment choice and considered that conservative treatment was less effective for
LSS, due to $\mathrm{LFH} ;{ }^{20}$ however, conflicting opinions regarding the surgical indications for LSS have been expressed. Most reports have indicated that the symptoms of patients with LSS, such as lower-extremity pain, intermittent claudication, and cauda equina syndrome, seriously affect activities of daily life and work. The overall recommendation is that surgical treatment should be performed in patients who have undergone conservative treatment for 3-6 months without significant remission and able to 

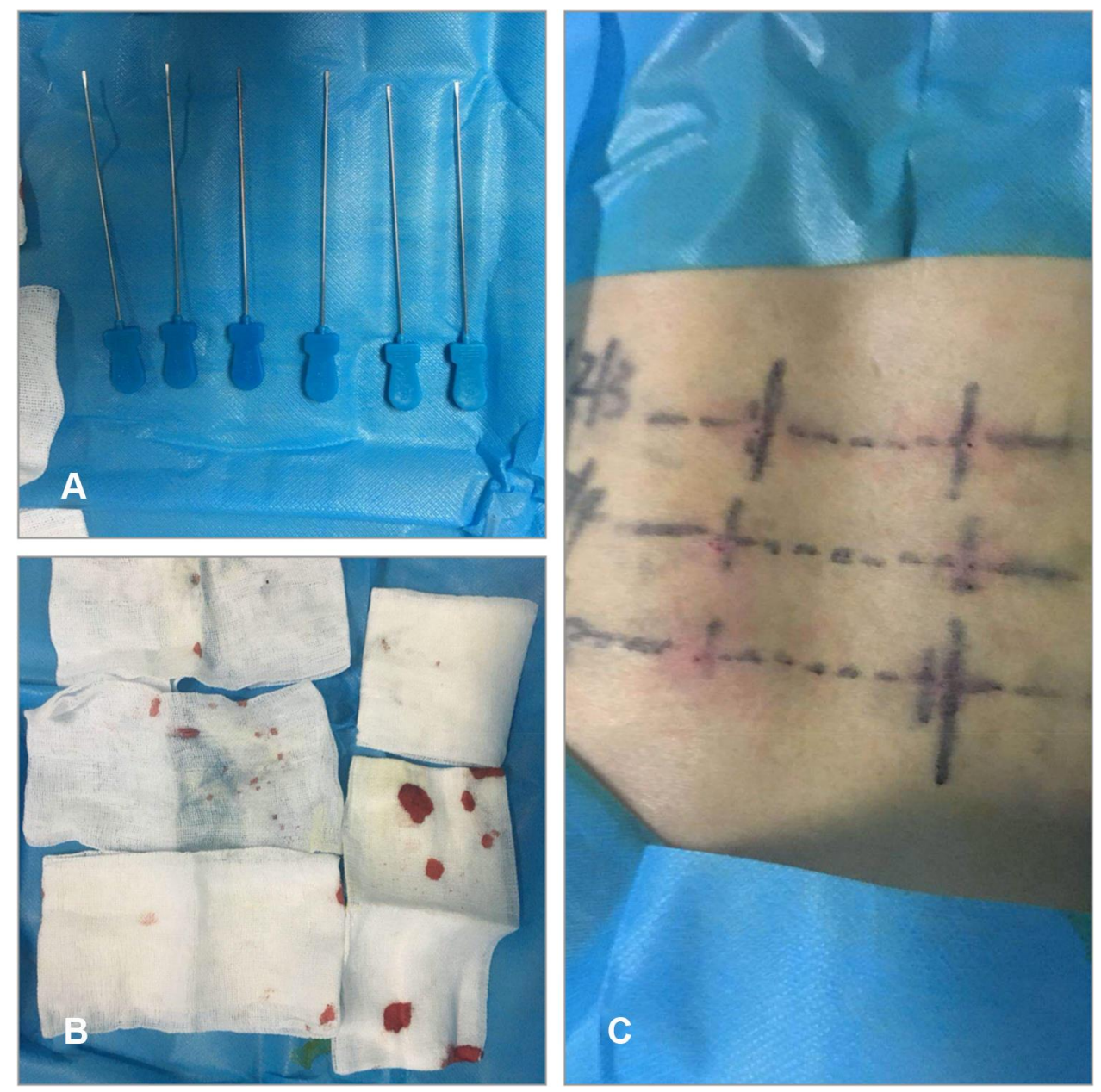

Figure 4 (A) The needle-knife used in the surgery; (B) intraoperative blood loss; (C) postoperative body-surface situation.

tolerate surgery, and the goal of the different treatment methods for degenerative LSS was to relieve the patient's symptoms. ${ }^{7}$ In general, LSS leads to slow regression of function, but is not life-threatening. Some patients' symptoms remain unchanged without deterioration or improvement of condition after a period, and conservative treatment is considered acceptable for some patients with mild LSS. Recently, minimally invasive surgery has become increasingly popular, due to low incidence of iatrogenic trauma, similar clinical outcomes compared to invasive operations, and shorter hospital stay.
In our study, we achieved successful treatment through needle-knife release of the lumbar LF. The patient's waist and leg pain were completely resolved immediately after the operation. Follow-up results at 1, 2, 4, 6, and 12 months after treatment showed that the short-term and long-term effects were stable (Table 2). The mechanism of action involves the cutting action of the needle knife to break off some of the LFH fibers, thereby reducing tension, pressure in the spinal canal, and stimulation of the nerve roots, and restoring biomechanical balance of the soft tissue. ${ }^{14}$ In the immediate postoperative period, we conducted CT and observed

Table I Preoperative and Postoperative Thickness of the Bilateral Ligamentum Flavum

\begin{tabular}{|c|c|c|c|c|c|c|}
\hline & \multicolumn{2}{|l|}{$L 2 / 3$} & \multicolumn{2}{|l|}{ L3/4 } & \multicolumn{2}{|l|}{ L4/5 } \\
\hline & Before surgery & After surgery & Before surgery & After surgery & Before surgery & After surgery \\
\hline Left & 0.4 & 0.4 & 0.5 & 0.5 & 0.7 & 0.6 \\
\hline Right & 0.6 & 0.5 & 0.6 & 0.5 & 0.7 & 0.6 \\
\hline
\end{tabular}


Table 2 Preoperative and Follow-Up Results of VAS and JOA

\begin{tabular}{|l|l|l|}
\hline & VAS & JOA \\
\hline Preoperative & 6 & 10 \\
Postoperative I month & 0 & 29 \\
Postoperative 2 months & 0 & 29 \\
Postoperative 4 months & 0 & 29 \\
Postoperative 6 months & 0 & 29 \\
\hline
\end{tabular}

Table 3 Literature Review

\begin{tabular}{|l|l|}
\hline & $\mathbf{5 4 0}$ Cases \\
\hline Average age, years & $54.2(33-79)$ \\
Sex (female/male) & $166 / 374$ \\
Excision of the ligamentum flavum & $194(35.9 \%)$ \\
Decompressive laminectomy & $44(8.1 \%)$ \\
Minimally invasive decompression & $149(27.6 \%)$ \\
Epidural steroid injections & $153(28.4 \%)$ \\
\hline
\end{tabular}

reduced thickness of the LF compared to before surgery (Table 1).

The needle knife is a type of surgical treatment using a fine scalpel, was introduced byHanzhang Zhu in the 1970s, and combines traditional Chinese medicine with modern Western medicine. Since its inception, it has been widely used in traditional Chinese-medicine hospitals and "barefoot doctor" clinics in China (Figure 4). Percutaneous lumbar LF release by needle knife has gained popularity for the treatment of lumbar disk herniation and other conditions affecting this area. However, applicability of this method in patients with LSS remains unclear. Usually, in acupotomy (Figures 5 and 6), the part to be treated must be stripped and peeled four or five times to form the same cut trace of a discontinuous line. Such an operation can form a row of small holes in the thickened LF and bone attachment to decompress and repair. The diameter of the acupuncture needle used in acupotomy is usually $0.4-1.2 \mathrm{~mm}$, similar to the size of a syringe needle. Therefore, acupotomy has the shovel and lysis effect from the insertion and puncture operation in human tissue that the human body can basically repair spontaneously, with an extremely low possibility of scar formation. The difference between the lysis in acupotomy and the complete breaking of the fiber in surgical operation is that the former belongs to a local fiber disconnection and the human body can perform the repair mechanism after the operation. Following LFacupotomy lysis, the abnormal stress caused by LFH will be alleviated to a certain extent, which decompresses the posterior of the spinal canal and reduces its compression of the dural sac and nerve root.

In this case, the imaging results of the lumbar spine contributed less to making diagnosis than the symptoms. Use of imaging findings alone may have led to the decision to pursue conservative treatment, and as a consequence seriously impacted the patient's quality of life. Treatment of degenerative LSS should consider the holistic concept and target the bone structure, soft-tissue balance, and lumbar back muscle's fascia chain. Such an approach achieves alleviation of symptoms compared to processing based on the results of imaging examination alone. $^{22}$ Early evaluation of the patient's course, symptoms, and signs would allow early diagnosis and treatment with minimum cost and maximum therapeutic effect. Individualized diagnosis and treatment plans for each patient involving gradual stepwise treatment using conservative minimally invasive surgery and segmentation show
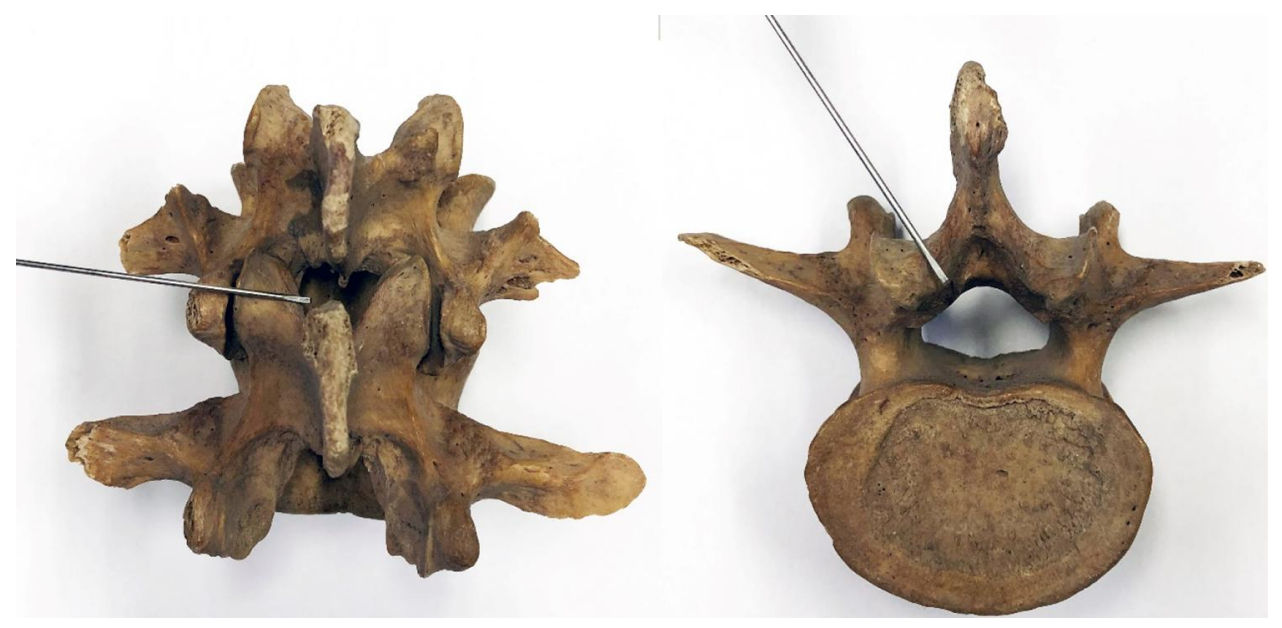

Figure 5 Needle-knife insertion into the yellow ligament inside the facet joint. 


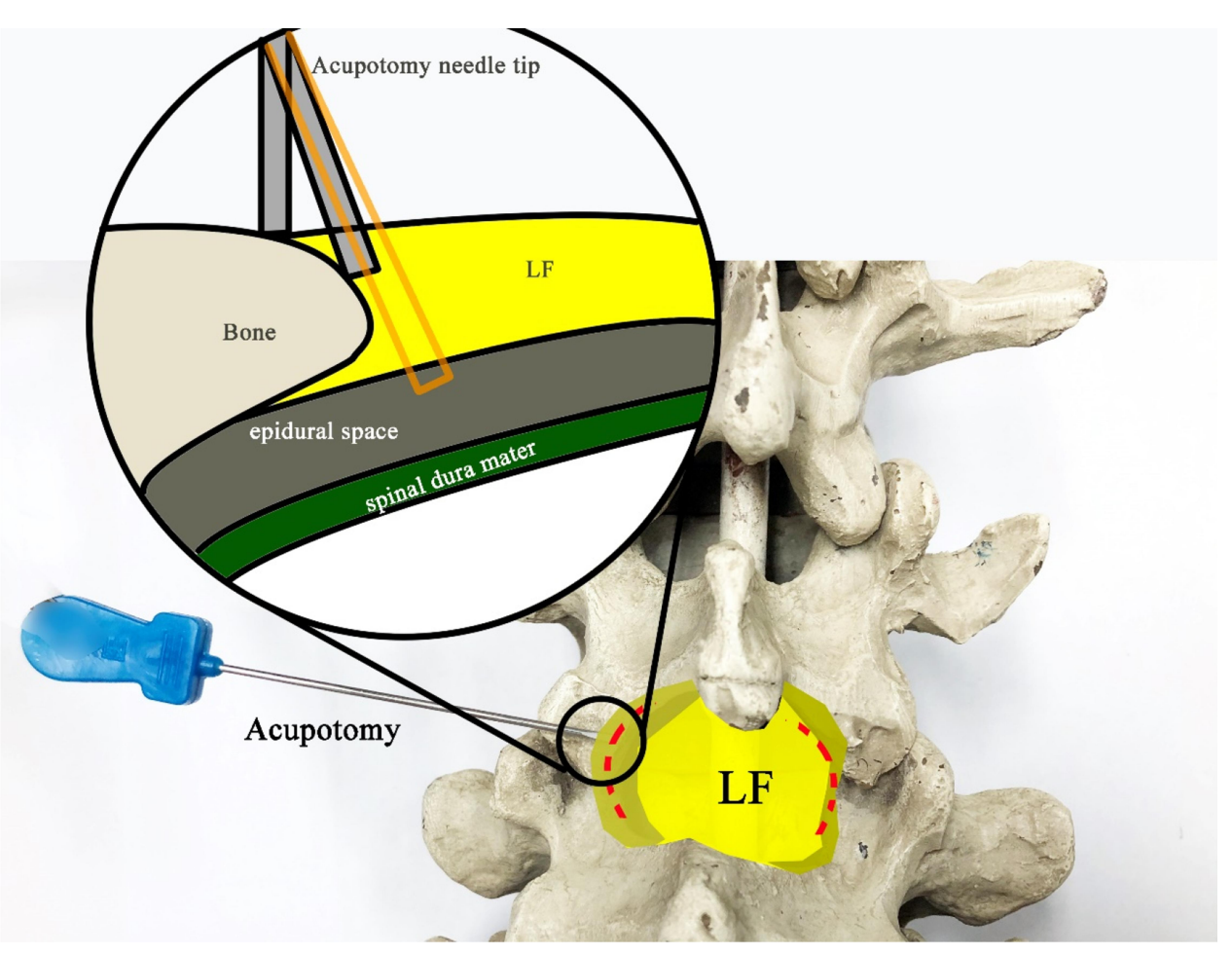

Figure 6 The needle-knife area stripped and peeled four or five times to form the cut trace of a discontinuous line and needle-tip operation on the bone surface without a feeling of falling to avoid puncturing the spinal dura mater. LF, ligamentum flavum. Orange rectangle: needle knife is not in the bone surface.

potential to prevent excessive surgery, minimize complications, and ensure effectiveness and safety during treatment.

In conclusion, needle-knife therapy is a minimally invasive treatment of traditional Chinese medicine in China. We conducted needle-knife therapy under CT guidance to ensure accuracy and safety of treatment in a patient with degenerative LSS. Advancements in minimally invasive surgery would allow the development of lumbar surgery in future. This report of our experience with CT-guided percutaneous lumbar LF release by needle knife in the treatment of LSS has potential for use as a clinician's reference.

\section{Ethics}

The patient signed the written informed consent, including that the case details and any accompanying images can be published, except for personal information. This manuscript did not need institutional approval to publish the case details.

\section{Author Contributions}

$\mathrm{XZ}$ and $\mathrm{ZQ}$ made the same contribution to this work and are co-first authors. All authors made substantial contributions to conception and design, acquisition or analysis and interpretation of data, took part in drafting the article or revising it critically for important intellectual content, gave final approval to the version to be published, and agree to be accountable for all aspects of the work. All authors read and approved the final manuscript.

\section{Disclosure}

Xinyue Zhu and Zuyun Qiu are co-first authors for this study. The authors report no conflicts of interest in this work.

\section{References}

1. Lee SY, Kim T-H, Oh JK, et al. Lumbar stenosis: a recent update by review of literature. Asian Spine J. 2015;9(5):818-828. doi:10.4184/ asj.2015.9.5.818

2. Deyo RA. Treatment of lumbar spinal stenosis: a balancing act. Spine J. 2010;10(7):625-627. doi:10.1016/j.spinee.2010.05.006

3. Benoist M. The natural history of lumbar degenerative spinal stenosis. Joint, Bone, Spine: Revue Du Rhumatisme. 2002;69(5):450-457. doi:10.1016/S1297-319X(02)00429-3

4. Arbit E, Pannullo S. Lumbar stenosis: a clinical review. Clin Orthop Relat Res. 2001;384:137-143. doi:10.1097/00003086-200103000-00 016

5. Ammendolia C, Stuber K, Tomkins-Lane C, et al. What interventions improve walking ability in neurogenic claudication with lumbar spinal stenosis? A systematic review. Eur Spine J. 2014;23(6):1282-1301. doi:10.1007/s00586-014-3262-6

6. Ammendolia C, Stuber K, De Bruin LK, et al. Nonoperative treatment of lumbar spinal stenosis with neurogenic claudication: a systematic review. Spine. 2012;37(10):E609-16. doi:10.1097/BRS.0b013e3182 $40 \mathrm{~d} 57 \mathrm{~d}$ 
7. Kalichman L, Cole R, Kim DH, et al. Spinal stenosis prevalence and association with symptoms: the Framingham Study. Spine J. 2009;9 (7):545-550. doi:10.1016/j.spinee.2009.03.005

8. Deyo RA, Gray DT, Kreuter W, et al. United States trends in lumbar fusion surgery for degenerative conditions. Spine. 2005;30(12):1441-5; discussion 6-7. doi:10.1097/01.brs.0000166503.37969.8a

9. Parker SL, Godil SS, Mendenhall SK, et al. Two-year comprehensive medical management of degenerative lumbar spine disease (lumbar spondylolisthesis, stenosis, or disc herniation): a value analysis of cost, pain, disability, and quality of life: clinical article. J Neurosurg Spine. 2014;21(2):143-149. doi:10.3171/2014.3.SPINE1320

10. Harrop JS, Hilibrand A, Mihalovich KE, et al. Cost-effectiveness of surgical treatment for degenerative spondylolisthesis and spinal stenosis. Spine. 2014;39(22 Suppl 1):S75-85. doi:10.1097/BRS.00 00000000000545

11. Deyo RA, Mirza SK, Martin BI, et al. Trends, major medical complications, and charges associated with surgery for lumbar spinal stenosis in older adults. JAMA. 2010;303(13):1259-1265. doi:10.10 01/jama.2010.338

12. Mizuno J, Nakagawa $H$, Inoue $T$, et al. Ligamentum flavum hematoma in the lumbar spine. Neurol Med Chir (Tokyo). 2005;45 (4):212-215. doi:10.2176/nmc.45.212

13. Sun XZ, Chen ZQ, Qi Q, et al. Diagnosis and treatment of ossification of the ligamentum flavum associated with dural ossification: clinical article. J Neurosurg Spine. 2011;15(4):386-392. doi:10.31 71/2011.5.SPINE10748

14. Yoshiiwa T, Miyazaki M, Kawano M, et al. Analysis of the relationship between hypertrophy of the ligamentum flavum and lumbar segmental motion with aging process. Asian Spine J. 2016;10 (3):528-535. doi:10.4184/asj.2016.10.3.528
15. Saito T, Yokota K, Kobayakawa K, et al. Experimental mouse model of lumbar ligamentum flavum hypertrophy. PLoS One. 2017;12(1). doi:10.1371/journal.pone.0169717.

16. Chelladurai A, Balasubramaniam S, Anbazhagan SP, et al. Dorsal spinal ligamentum flavum thickening: a magnetic resonance imaging study. Asian Spine J. 2018;12(1):47-51. doi:10.4184/asj.2018.12.1.47

17. Kang KC, Lee CS, Shin SK, et al. Ossification of the ligamentum flavum of the thoracic spine in the Korean population. $J$ Neurosurg Spine. 2011;14(4):513-519. doi:10.3171/2010.11.SPINE10405

18. Altinkaya N, Yildirim T, Demir S, et al. Factors associated with the thickness of the ligamentum flavum: is ligamentum flavum thickening due to hypertrophy or buckling? Spine. 2011;36(16):E1093-7. doi:10.1097/BRS.0b013e318203e2b5

19. Park JO, Lee BH, Kang YM, et al. Inflammatory cytokines induce fibrosis and ossification of human ligamentum flavum cells. J Spinal Disord Tech. 2013;26(1):E6-12. doi:10.1097/BSD.0b013e318269 8501

20. Ergun T, Lakadamyali $H$. The relationship between the prevalance and size of lumbar ossified ligamentum flavum and the presence and degree of facet joint degeneration. Eur $J$ Radiol. 2012;81 (11):3456-3460. doi:10.1016/j.ejrad.2012.05.009

21. Tomkins-Lane $\mathrm{C}$, Melloh M, Lurie J, et al. ISSLS prize winner: consensus on the clinical diagnosis of lumbar spinal stenosis: results of an international Delphi Study. Spine. 2016;41(15):1239-1246. doi:10.1097/BRS.0000000000001476

22. Prommahachai A, Wittayapirot K, Jirarattanaphochai $K$, et al. Correction with instrumented fusion versus non-corrective surgery for degenerative lumbar scoliosis: a systematic review. J Med Assoc Thailand $=$ Chotmaihet Thangphaet. 2010;93(8):920-929.
Journal of Pain Research

\section{Publish your work in this journal}

The Journal of Pain Research is an international, peer reviewed, open access, online journal that welcomes laboratory and clinical findings in the fields of pain research and the prevention and management of pain. Original research, reviews, symposium reports, hypothesis formation and commentaries are all considered for publication. The manuscript management system is completely online and includes a very quick and fair peer-review system, which is all easy to use. Visit http:// www.dovepress.com/testimonials.php to read real quotes from published authors. 